\title{
The Development of Explanation of Falling Cat Problem and Applications of Falling Cat Phenomenon
}

\author{
Junhua Yang, \\ ${ }^{1}$ Suzhou Foreign Language School, Suzhou, China, 215000 \\ *Corresponding author. Email: Yangjunhua2022@163.com
}

\begin{abstract}
As a basic law in mechanics, the principle of conservation of angular momentum illustrates that angular momentum of a system is conserved until it is acted by an external force. It is difficult for people who are falling down to change his or her orientation. However, cats can complete the change of orientation and land on their feet even though released from rest upside down. It is named Falling Cat Problem. The purpose of the paper is to analyse whether the falling cat phenomenon violates the principle of conservation of angular momentum. It also includes some related research in the past. The result is that the falling cat phenomenon obeys the principle of conservation of angular momentum. In addition, the paper introduces some applications of the falling cat phenomenon in our real life.
\end{abstract}

Keywords: Falling Cat Problem, conservation of angular momentum, T.R.Kane, application of falling cat phenomenon

\section{INTRODUCTION}

People who have a pet cat may have found that cats can complete the change of orientation and land on their feet even though released from rest upside down. The falling motion of cats seems to violate the principle of conservation of angular momentum, which troubled scientists for many years. People wondered if there was any factors facilitating the success of cats' falling. Many scientists contribute to the explanation of the falling cat problem. French scientist Étienne-Jules Marey invented the first chronophotographic gun and filmed the successive segments of falling motion of cats for the first time. The Theoretical Mechanics Course published in 1955 proposed the tail spinning theory. In 1969, Kane published his paper A Dynamical Explanation of Falling Cat Phenomenon.

Through summarizing some relevant literatures, this paper includes how scientists approaches the most reasonable answer, simple analysis of the falling cats problem and some applications in real life. The falling cat phenomenon is not a popular topic - most people don't even notice it although it happens every day in our life. Anyhow, it is widely applied nowadays in our life.

\section{EXPLORATION OF THE CATS' FALLING}

In 1882, French scientist Étienne-Jules Marey invented the first chronophotographic gun and first filmed the falling motion of cats. Chronophotographic gun is an instrument that could take 12 consecutive photos per second. During the experiment, a cat was held by its feet and let go to fall to the ground. The chronophotographic gun snapped the successive process of the cat's falling and humans could observe the division of the falling of cats for the first time (Figure 1). Marey's research was published in Nature. Marey also published his investigations of the motion of falling cats in the prestigious French Proceedings of the Academy of Sciences. He concluded that it was the inertia of the cat's own mass that enabled it to right itself, using separate actions of its muscles in its front and rear halves [1]. 


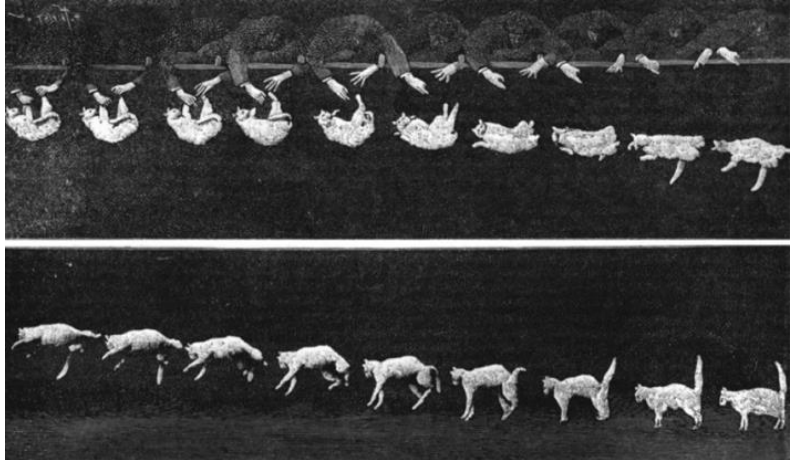

Figure 1 Successive images from 1894 of a falling cat were captured in photographs taken at a rate of 12 frames per second with a "chronophotographic gun." [2]

The Theoretical Mechanics Course published in 1955 states that it is rotating their tails that facilitates cats to turn around while falling. If a cat spins its tail quickly enough, it can complete the motion of rotating its body in an opposite direction. The conclusion was compiled into many textbooks at that time. However, the tail spinning theory was later been proved incorrect. British Physiologist D. A. McDonald used a rumpy to carry out the similar experiment and discovered that the rumpy could also spin their body. As a result, the tail spinning theory was rejected fundamentally. From people's common sense, a cat's tail is very light and takes only about $3 \%$ of its whole weight. Thus, according to the tail spinning theory, a cat needs to turn its tail several rounds in a few seconds to rotate its body for 180 degrees in order to make angular momentum conserved. It is illogical for cats to spin their tail Uber such frequency and people did't find related evidence by observing the falling motion of cats. Until 1969, Kane from Stanford University finally gave a theoretical explanation about the falling cat phenomenon in his paper A Dynamical Explanation of Falling Cat Phenomenon.

\section{SIMPLE EXPLANATION OF THE FALLING CAT ISSUE}

There are countless explanations for the falling cat phenomenon. Some have been proved wrong, while some are quite convincing and seems to be the final answer. However, in real life, people needs to consider a lot of factors that may influence the falling motion of a cat, such as the torsion of the backbone and some environment factors like the wind. The explanation below is to illustrate that the falling cat phenomenon obeys the principle of conservation of angular momentum. There are only simple theoretical analysis without specific research and data of the motion of falling cats and some skills that cats may use, which is based on knowledges of rotational mechanics.

People can utilize modeling to analyse the falling cat problem. A cat needs to bend its body at first when falling. People can view the the front part and the hind part of its body as two rigid cylinders with different rotational axes. The two parts of the cat's body both rotate in a cone. Moreover, angular momentum of the two parts are counteracted, which means the resultant angular momentum is 0 . This follows the principle of conservation of angular momentum. It is because the cat's hind part spins in the opposite direction of the front part does.

Skaters retract their arms to spin faster and stretch out their arms to lower down spinning. Similarly, there are also some skills useful for cats to make their turning easier. Observing a cat's falling in detail, people can find that the cat first retracts its front legs and straightens its back legs. This phenomenon can be explained by the change of rotational inertia. Rotational inertia is a property of any object which can be rotated. It is a scalar value which tells us how difficult it is to change the rotational velocity of the object around a given rotational axis.[3] The symbol of rotational inertia is I.

$$
\mathrm{I}=\int \mathrm{r}^{2} \mathrm{dm}(1)
$$

Thus, the rotational inertia of the front body decreases when the cat retracts its front legs while the rotational inertia of the hind body increases when the cat straightens its back legs.

$$
\mathrm{L}=\mathrm{I} \omega, \omega=\mathrm{d} \theta / \mathrm{dt}(2)
$$

While rotational inertia decreases, the angular velocity will increases since the angular momentum is constant. Similarly, while rotational inertia increases, the angular velocity will decreases. As a result, in order to rotate its front body 180 degrees clockwise, a cat may only need to spin its hind body about 20 degrees anticlockwise. In the same way, the cat will straighten its front legs and retract its back legs to turn the hind body for 180 degrees.

The analyses above show that the cat falling isssue follows the principle of conservation of angular momentum. Furthermore, there are more accurate and detailed explanation for cat falling phenomenon. In reality, there are more factors to consider, such as the motion of the backbone of a falling cat.

\section{KANE'S EXPLANATION ABOUT CAT FALLING PROBLEM}

Observing photographs of cats as they fell, Kane found three characteristics of cats' movement:

(1) The spine is curved, but there is no mutual torsion between different parts

(2) The spine from the forward curve is along a certain direction to lateral bending, backward bending, lateral bending, and then forward bending to the initial bending state

(3) The degree of posterior curvature of the spine is much less than that of anterior curvature; [4] 
These motor characteristics are not directly consistent with the previous explanation, and Kane modified them by suggesting that cats fall with their spines bending in all directions (front, right, back, left or front, left, back, right), much like the conical movement of the waist in gymnastics. When the front part of a cat moving like this, the cat's whole body will rotate in the opposite direction, and when the front half cone moves for one period, the whole body will rotate in the opposite direction of $180^{\circ}$. In order to verify the correctness of this theory, Kane established A physical model of the cat body in Figure 2, with two cylinders A and B represented respectively. The front and back parts of the cat are shown, and the two cones in the middle are used for rolling without sliding to simulate the constraint condition of bending without twisting described above. He listed differential equations of motion for two rigid body systems and carried on computer numerical simulation. The calculation results are output by graph in Figure 3. The positive signs indicate the cat's limbs, and the black dots between the columns indicate where the cylinders meet, which is the direction of the curvature of the backbone.

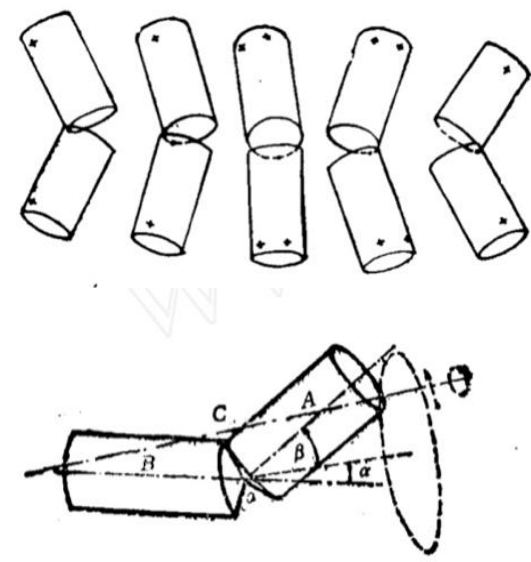

Figure 2 Modeling of a falling cat (viewing the cat's front part and hind part of the body as two rigid cylinders) [6]

\section{APPLICATIONS RELATED TO FALLING CAT PROBLEM IN REAL LIFE}

Kane's research shows that any object can rotate without help of external force, which attracts NASA's attention. Supported by NASA, Kane researched on the skills that astronauts can use to turn around when experiencing weightlessness. He found a trampoline athlete, who had a flexible body, to imitate cats' motion in a spacesuit. The picture was posted on Life in 1968 (Figure 3). Nowadays, imitating a cat to turn around in space is one of the basics for astraunaut.

Furthermore, the change of direction of Harbour telescope is also related to the secret of falling cat phenomenon. It is unpractical to change the face by emitting gas. Then scientists apply the falling cat phenomenon into the design of Harbour telescope. In 2009, during the final servicing mission, all six of Hubble's gyroscopes were replaced. The gyroscopes (Figure 4) maintain orientation and provide stability by pushing back against any force that attempts to change its orientation.[6]

Last but not the least, falling cat phenomenon also have reflections in the microscopic world. Scientists show that a five-bead polymer chain can change its orientation about its longitudinal axis largely, e.g., 120。, under conditions of zero total angular momentum by changing the two dihedral angles in a cyclic manner. This phenomenon is an example of the so-called "falling cat" phenomenon, where a falling cat undergoes a geometric somersault by changing its body shape under conditions of zero total angular momentum. [7]

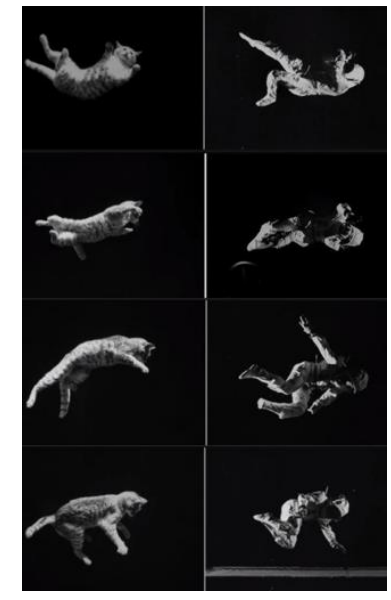

Figure 3 Photographs of an "astronaut" imitating a cat's falling motion [8]

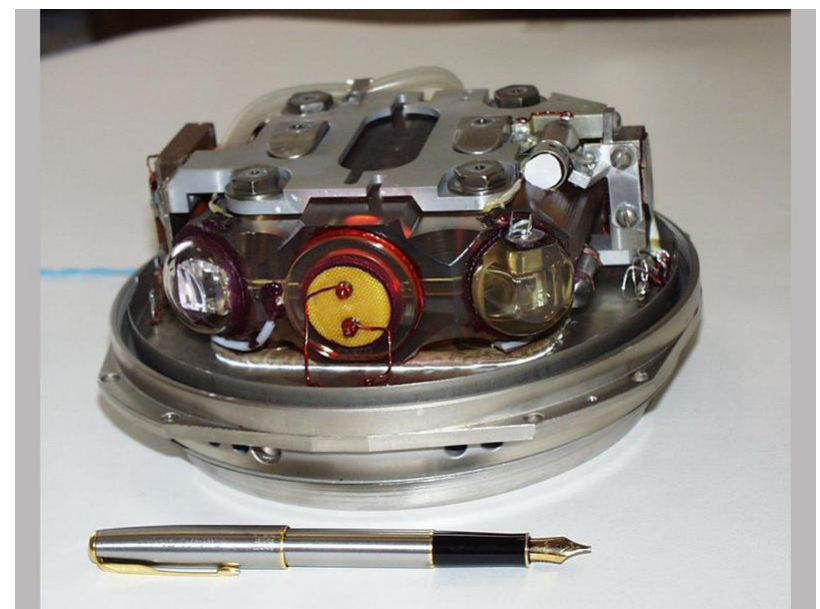

Figure 4 A super-precise laser gyroscope was developed by the Russian scientific research and design[9]

\section{DISCUSSION}

According to some literature and theoretical summaries, the paper includes the exploration of the cat's falling, simple explanation of the falling cat problem, 
Kane's explanation about the falling cat problem, and the applications related to the falling cat problem in our daily life. It concludes that the falling cat phenomenon obeys the principle of angular momentum. There are some skills for cat's turning which can be explained by the principle of conservation of angular momentum. However, because of lack of time and proper devices, the essay doesn't analyse specific data. If there is proper research and data, the explanation will be more convincing and clear.

\section{CONCLUSION}

Scientists have been interested in the falling cat problem since the nighteenth century because they found turning around without external force unscientific. The effort paid by these scientists all contributes to the answer how the cat falling problem obeys the principle of conservation of angular momentum. Moreover, the falling cat phenomenon inspires many designs in our daily life. For instance, the falling cat phenomenon is applied in the turning of astronauts and the change of direction of Harbour Telescope. Moreover, it also has a reflection in the microscopic world. It is fanscinating that people can find connections between the microscopic world and the microscopic world from time to time and physics principle is like the bridge between them. Although a lot of scientists have resaerched on the falling cat phenomenon, there are still a lot worthwhile to learn about it. In conclusion, simple and usual phenomena can also be objects to research in detail since they sometimes can trigger questions difficult to answer. People should not omit things in our daily life and should think about how these principles can be used to raise the quality of our life.

\section{AUTHORS' CONTRIBUTIONS}

This paper is independently completed by Junhua Yang.

\section{ACKNOWLEDGMENTS}

During the process of writing the paper, my professor, my teaching assistant, my thesis advisor and my parents provide me with a lot of support. Firstly, thank my professor and my teaching assistant for teaching me professional knowledges and giving me suggestions about the topic of my paper. When I have academic problems with my paper, I always go to them. Secondly, thank my thesis advisor for instructing me to write a independent essay and give me some correcting advices patiently. Last but not least, thank my parents for encouraging me to do the subject and write an independent essay for the first time. Though it is quite challenging for a high school student, I manage to complete it for my best under the spiritual handholds from my parents.

\section{REFERENCES}

[1] Howard Wainer and Michael Friendly, American scientist: falling cats and photography guns, 2019 July-August, p211.

[2] Étienne-Jules Marey, Photography of the successive falling motion of a cat filmed by a chronophotographic gun, 1894.

[3] Jia shuhui, The turning motion of cats and sports biomechanics, China Academic Journal Electronic Publishing House, 1994-2006.

[4] T. R. Kane, A dynamical explanation of the falling cat phenomenon, Int. J. Solids Structures. 1969. Vol. 5. pp. 663 to 670. Pergamon Press. Printed in Great Britain.

[5] T. R. Kane, Figure from A dynamical explanation of the falling cat phenomenon, Int. J. Solids Structures. 1969. Vol. 5. pp. 663 to 670. Pergamon Press. Printed in Great Britain.

[6] Ethan Siegel and Starts With A Bang, This is how Hubble will use its remaining gyroscopes to maneuver in space, 2018. https://www.forbes.com/sites/startswithabang/2018 /10/16/this-is-how-hubble-will-use-its-remaining-g yroscopes-to-maneuver-in-space/?sh=796275152ba 8.

[7] Tomohiro Yanao and Taiko Hino, Geometric somersaults of a polymer chain through cyclic twisting motions, Department of Applied Mechanics and Aerospace Engineering, Waseda University, Tokyo January 23, 2017:169-8555.

[8] Life, photography of a falling cat and an astronaut, 1968.

[9] Ethan Siegel and Starts With A Bang, This is how Hubble will use its remaining gyroscopes to maneuver in space, picture A super-precise laser gyroscope was developed by the Russian scientific research and design, 2018 . https://www.forbes.com/sites/startswithabang/2018 /10/16/this-is-how-hubble-will-use-its-remaining-g yroscopes-to-maneuver-in-space/?sh $=796275152$ ba 8. 